\title{
Induction of Fos protein immunoreactivity by spinal cord contusion
}

E.A. Del-Bel ${ }^{3}$,

C.A.G. Borges ${ }^{1}$,

H.L.A. Defino ${ }^{1}$ and F.S. Guimarães ${ }^{2}$

\author{
D epartamentos de ${ }^{1}$ Cirurgia, O rtopedia e Traumatologia, H ospital das Clínicas, \\ ${ }^{2}$ Farmacologia, Faculdade de Medicina de Ribeirão Preto, and \\ ${ }^{3} \mathrm{M}$ orfologia, Estomatologia e Fisiologia, Faculdade de 0 dontologia de Ribeirão Preto, \\ Universidade de São Paulo, Ribeirão Preto, SP, Brasil
}

\section{Correspondence \\ E.A. Del-Bel \\ Departamento de Morfologia, \\ Estomatologia e Fisiologia \\ FO RP, USP \\ 14040-904 Ribeirão Preto, SP \\ Brasil \\ Presented at the International Neurobiology Course, Faculdade de $O$ dontologia de Ribeirão Preto, Universidade de São Paulo, Ribeirão Preto, SP, Brasil, May 20-27, 1998. \\ Part of a M aster's thesis presented by C.A.G. Borges to the Departa- mento de Cirurgia, O rtopedia e Traumatologia, Ribeirão Preto, SP. Research supported by FAPESP, CNPq and FAEPA.}

Received December 9, 1998 Accepted February 8, 2000

\section{Abstract}

The objective of the present study was to identify neurons in the central nervous system that respond to spinal contusion injury in the rat by monitoring the expression of the nuclear protein encoded by the $c$-fos gene, an activity-dependent gene, in spinal cord and brainstem regions. Rats were anesthetized with urethane and the injury was produced by dropping a 5-g weight from $20.0 \mathrm{~cm}$ onto the exposed dura at the T10-L1 vertebral level (contusion group). The spinal cord was exposed but not lesioned in anesthetized control animals (laminectomy group); intact animals were also subjected to anesthesia (intact control). Behavioral alterations were analyzed by Tarlov/ Bohlman scores, $2 \mathrm{~h}$ after the procedures and the animals were then perfused for immunocytochemistry. The patterns of Fos-like immunoreactivity (FLI) which were site-specific, reproducible and correlated with spinal laminae that respond predominantly to noxious stimulation or injury: laminae I-II (outer substantia gelatinosa) and X and the nucleus of the intermediolateral cell column. At the brain stem level FLI was detected in the reticular formation, area postrema and solitary tract nucleus of lesioned animals. No Fos staining was detected by immunocytochemistry in the intact control group. However, detection of FLI in the group submitted to anesthesia and surgical procedures, although less intense than in the lesion group, indicated that microtraumas may occur which are not detected by the Tarlov/ Bohlman scores. There is both a local and remote effect of a distal contusion on the spinal cord of rats, implicating sensory neurons and centers related to autonomic control in the reaction to this kind of injury.

The neurons of the mammalian central nervous system (CNS) respond to a lesion with an intense cell body reaction that may result in neuronal survival or death, with the acute post-injury phase being of critical importance $(1,2)$. The changes in blood flow and tissue perfusion that produce vascular

\section{Key words}

- Fos immunohistochemistry

- Intermediolateral column neurons

- Reticular formation

- Area postrema

- Dorsal horn

- Solitary tract nuclei

- Laminectomy

- Spinal cord injury damage around the site of the lesion, causing regional ischemia, may be responsible for the secondary lesion and consequent neuronal death (2). The damaging agent may produce physiopathological changes near the site of the lesion, as well as in brain centers functionally connected to the lesion or other 
damaged pathways $(3,4)$. However, little is known about the molecular mechanism that initiates cellular reaction after injury.

Among the molecular and cellular mechanisms that may contribute to the medium/long-term neuronal response are changes in gene expression $(4,5)$. Immediate early genes (IEG) are expressed transiently and rapidly in nerve cells by a broad array of stimuli. Among the best studied IEG are the Fos (c-fos, fos B, fra-1, fra-2) and c-jun (cjun, jun $B$, jun D) families. The expression of Fos protein in nerve cells is activity-dependent and related to the stimulus applied. In addition, the localization of the IEG may reflect a pattern of neuronal activation related to the type of stimulus and to the longterm variation in neuronal physiology $(4,6)$.

Axotomy of the CNS was reported to induce c-Fos immunoreactivity in the red nucleus, the locus coeruleus and motor cortex $(3,7)$. Recently, Ruggiero et al. (8) employed the Fos gene to monitor peripheral and central responses to spinal cord transection. Patterns of $c$-fos gene expression were site-specific and correlated with laminae that respond predominantly to noxious stimulation and contain sympathetic interneurons. Unfortunately, these studies do not mention the remote effects of a distal spinal cord lesion in brain stem nuclei correlated with autonomic control. Therefore, we investigated here the expression of Fos-like immunoreactivity (FLI), used as a marker of neural activity, at local and remote sites following spinal cord contusion injury.

Male Wistar rats weighing 250-300 g were used. Animals had free access to water and food and were kept on a 12-h light-dark cycle (lights on at 7:00 a.m.), under controlled temperature conditions. All experiments were performed between 9:00 and 12:00 a.m., and complied with the "Guide for the Use and Care of Animals" from the Brazilian Society of Neurosciences and Behavior. The site of lesion selected was the vertebral segment located between T10 and
L1. The animals were anesthetized with urethane $(25 \%, 2 \mathrm{ml} / \mathrm{kg})$, placed in a stereotaxic apparatus and submitted to the procedure. A total of 9 animals was used, divided into 3 groups. Group 1 (intact control, $\mathrm{N}=3$ ) consisted of similarly anesthetized unoperated rats; in group 2 (laminectomy group, $\mathrm{N}=3$ ), the spine was approached along the posterior surface and bilateral laminectomy of the T10-L1 segment was performed to expose the dura mater, which was left intact. In group 3 (contusion group, $\mathrm{N}=3$ ), the dura mater was exposed as described above and a lesion was produced by impact on the spine (9). To produce this lesion, a metal disk was placed on the meninges and a 5-g weight was dropped on it by free fall from a height of $20.0 \mathrm{~cm}$ inside a Teflon tube. After surgery, the muscular and subcutaneous layers and the skin were sutured and the animals were allowed to rest in individual boxes.

Sensorimotor function was evaluated in each animal $2 \mathrm{~h}$ after the experimental procedure for 5 min according to the scale proposed by Tarlov and modified by Bohlman (9). Grade zero indicates complete paraplegia without movements, grade I small articulate movements, grade II large movements, grade III indicates that the animal can stand on its feet, grade IV indicates that the animal can walk, and grade $\mathrm{V}$ indicates that the animal can walk on a plane with a 20 -degree inclination. No obvious signs of pain or stress were seen in any group.

After sensorimotor evaluation (after 2.05 $\mathrm{h}$ ), the rats were anesthetized with ip injections of $25 \%$ urethane $(2.5 \mathrm{ml} / \mathrm{kg})$ and transcardially perfused with saline followed by $4 \%$ paraformaldehyde, and the spinal cord was removed for histology and immunocytochemistry. The spinal contusion level was examined at autopsy. All spinal cord contusions were confirmed at the T10-L1 vertebral level. After removal, the brains and spinal cord were kept overnight in 30\% sucrose solution and 35- $\mu \mathrm{m}$ cryostat coronal sections were obtained and processed for 
Fos immunohistochemistry as described by Del Bel et al. (10). Briefly, tissue sections were successively washed and incubated for $40 \mathrm{~h}$ with the primary Fos antibody $(1: 2000$, Cambridge Research Biochemical, Cambridge, UK). Sections were then processed by the avidin-biotin immunoperoxidase method (Vectastain ABC kit, Vector Laboratories, Inc., Burlingame, CA, USA) and FLI was visualized by the addition of the chromogen 3,3'-diaminobenzidine (DAB, Sigma Chemical Co., St. Louis, MO, USA) and hydrogen peroxide. The slices were mounted on slides and coverslipped for microscopic observation. FLI could be visualized as a brown reaction product inside neuronal nuclei whose location was determined using the Paxinos and Watson atlas (11). Segments of the thoracic and lumbar spinal cord and regions of the brain stem were analyzed. Spinal laminae and brain stem nuclei were distinguished on Nissl-stained adjacent sections.

Tissue from the laminectomy and contusion groups was processed at the same time for immunohistological staining; tissue from the intact control group was processed separately, but together with an FLI-positive control (FLI was induced in rats with $2 \mathrm{mg} / \mathrm{kg}$ haloperidol, ip). Stained neuronal bodies were analyzed using a computerized image analysis system. Images were captured from slides using a Leika microscope and CCD camera, together with the National Institutes of Health Image software 9.0 (W. Rasband, National Institute of Mental Health). Fospositive neuronal bodies were counted from a fixed-size area belonging to the spinal cord (laminae I-II and X) and midbrain (reticular formation, solitary tract nuclei and area postrema), measured bilaterally. For each treatment, three to five sections from each animal ( $\mathrm{N}=3$ animals/group) were evaluated. A mean value for cell density in each region of the animal was then calculated. The statistical analysis was performed using the $t$-test, and differences were considered significant when $\mathrm{P}<0.05$.

The evaluation of sensorimotor function showed no evidence of spinal cord injury due to the procedure in animals of the group submitted to anesthetic alone or anesthesia and laminectomy, with scores of 4 or 5 on the Tarlov/Bohlman scale in both cases. The group submitted to the spinal cord contusion showed partial or total paraplegia and all animals scored 1 or 2 on the Tarlov/Bohlman scale.

No Fos protein expression was detected in the intact control group; however, FLI was detected in the laminectomy and contusion groups, mainly in the spinal segments located between $\mathrm{T} 10$ and L1. In the contusion group, FLI was detected around the central spinal cord canal, in lamina $\mathrm{X}$, in laminae I and II (Figure 1B and C) of the dorsal horn, and in laminae V, VI, VII VIII, and IX/accessory nucleus of the ventral horn of the rat spinal cord. In these animals, this FLI pattern was also observed in thoracic segments of the spinal cord above the level of injury, with the same intensity. However, in the laminectomy group we observed some FLI in laminae V-VIII, IX/accessory nucleus of the ventral horn of the rat spinal cord, but only around the surgery site. In contrast, there was no Fos protein expression in laminae I or II (Figure 1C), either locally or outside the surgical site (upper thoracic level). The detection of FLI in the laminectomy group indicated that some microtraumas may have occurred during the surgical procedure, as supported by macroscopic signs of tissue damage.

Although the presence of FLI was observed in both the laminectomy and contusion groups, its levels and molecular distribution in the spinal cord were higher in the contusion group (Figure 1A,B; Figure 2D,E and F).

Distinct cholinergic cell clusters form the intermediate region of the thoracolumbar spinal cord and contain sympathetic preganglionic neurons (12). Interestingly, 

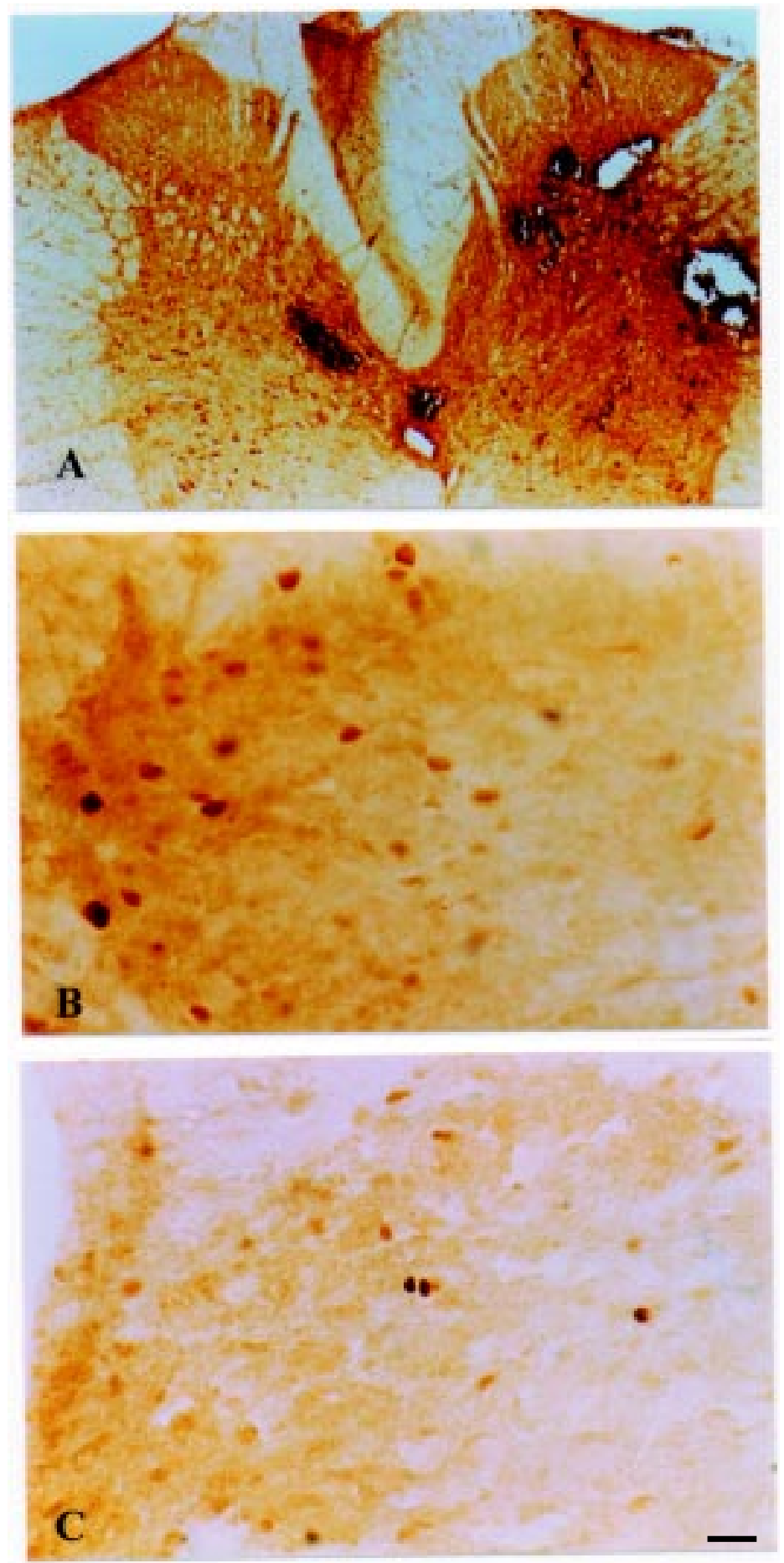

Figure 1 - Expression of Fos protein detected by immunocytochemistry in the thoracic segment of the spinal cord and in midbrain structures. A, Fos expression detected the lumbar segment level in group 3 animals (contusion). B, Fos expression detected in laminae I and II of the lumbar spinal cord in group 3 animals (contusion); detail of a sensory dorsal horn. C, Fos expression detected in laminae I and II of the lumbar spinal cord in group 2 animals (laminectomy); detail of the sensory dorsal hom. (A, Bar $=200 \mu \mathrm{m} ; \mathrm{B}, \mathrm{C}$, bar $=100$ $\mu \mathrm{m})$ neuronal cell bodies and processes of the principal intermediolateral nucleus, a welldefined neuronal population located in the lateral horn along the borderline between white and gray matter, stained intensely for Fos-immunoreactivity in the contusion group (Figure 2B), but not in either control group (Figure 2A).

In addition, FLI occurs in some brain stem centers involved in central autonomic control such as the ventrolateral reticular area of the medulla oblongata (Figure 2E), the area postrema and the solitary tract nucleus (Figure 2F). Less intense FLI expression was observed in tissues from the laminectomy group in the same areas (Figure $2 \mathrm{~B}$ and $\mathrm{C}$, respectively). No FLI was observed in the intact control group.

Differences in intensity of immunostaining between experimental and control animals were striking and clear cut. However, quantitative analysis of the number of FLI cells in the laminae of the spinal cord and midbrain nucleus shows that spinal cord contusion produced a large and consistent pattern of FLI cells (Table 1), with significant differences between the contused group and the laminectomy group in laminae I-II and X of the spinal cord, solitary tract nucleus, area postrema and nuclei of the reticular formation in the medulla oblongata $(\mathrm{P}<0.05)$.

The contusion injury produced by this procedure is heterogeneous (Figure 1A), a fact that should be considered in the analysis of the results (13). The impact zone contained bleeding and tissue damage; immunocytochemistry showed local expression of FLI $2 \mathrm{~h}$ after experimental spinal cord contusion; a less intense expression was seen in rats submitted to laminectomy. The topographic distribution of neurons containing FLI was not observed in intact control animals.

The precise mechanisms by which the effects of injury are distributed throughout the cord are unknown, but similar evidence was found by Yerzierski et al. (14), Thomp- 

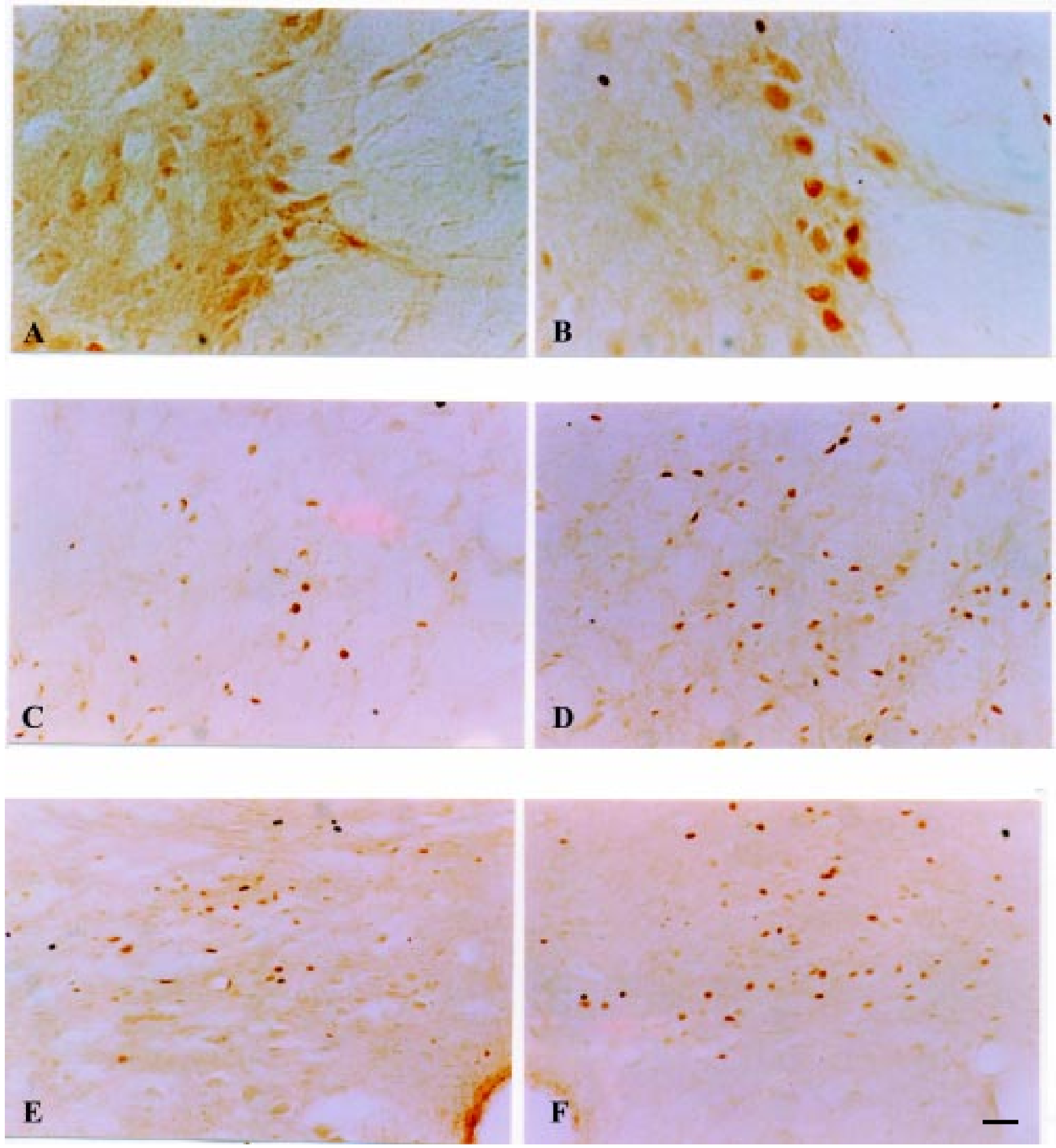

Figure 2 - Expression of Fos protein detected by immunocytochemistry in the thoracic segment of the spinal cord and in midbrain structures. A, Fos expression detected at the thoracic segment level in group 2 animals (laminectomy), in cells of the intermediolateral spinal cord nuclei. B, Fos expression detected at the thoracic segment level in group 3 animals (contusion), in cells of the intermediolateral spinal cord nuclei. C, Fos expression detected in nuclei of the reticular formation in group 2 animals (laminectomy). D, Fos expression detected in nuclei of the reticular formation in group 3 animals (contusion); E, Fos expression detected in the solitary tract nucleus at the midbrain level in group 2 animals (laminectomy). F, Fos expression detected in the solitary tract nucleus at the midbrain level in group 3 animals (contusion). Bar $=50 \mu \mathrm{m}$. 
son et al. (15) and Ruggiero et al. (8). Anesthesia is not thought to affect Fos expression; the results showed an effect of surgical stress, but the greater expression of Fos protein following spinal cord contusion suggests that spinal cord injury causes an increase in Fos expression.

The detection of Fos protein in animals submitted only to laminectomy supports the evidence that microtraumas of the spinal cord may occur during surgery. These microtraumas were not reflected in behavioral tests using the Tarlov/Bohlman scale for sensorimotor analysis. Sensitive behavioral tests should be tried in order to evaluate the functional importance of these minor injuries.

FLI expression presented a different pattern of distribution at the spinal cord laminar level in Rexed's laminae I-II of the dorsal horn, only in the animals submitted to contusion injury, in agreement with previous results (8). Yerzierski et al. (14) described an increased excitability in response to mechanical stimuli, elevated levels of background activity and long afterdischarge response after excitotoxic spinal cord injury. The functional changes and location of these cells support the importance of neurons in the superficial laminae of the injured cord for long-term physiological changes after contusion injury.

In animals submitted to spinal cord in- jury, Fos protein was also detected in the nuclei of the intermediolateral spinal cord that regulate the autonomic nervous system, and at higher levels (reticular formation of the brain stem, area postrema and solitary tract nuclei). These results suggest that the injury can affect (bilaterally) segments far away from the site of contusion. This is not surprising given the complexities of propriospinal connections and the likely involvement of propriospinal circuits in the distribution of the descending influences on the bulbospinal pathway. The nuclei of the autonomic nervous system of the reticular formation in which Fos protein was detected after impact injury are involved in blood pressure regulation, which is altered in tetraplegic and paraplegic patients (2). Cells of the intermediolateral column represent preganglionic sympathetic fibers that emerge via the ventral roots and project to various sympathetic ganglia. Krens and Weaver (16) suggested that the increased response to sensory stimulation below the level of spinal cord injury may result in an increase in muscle tonus and aberrant movements, elevation of blood pressure accompanied by headache, convulsive seizures and even a cerebrovascular accident. According to these investigators, these alterations may not result exclusively from the absence of descending inhibition of motor and autonomic neurons, but may also be under the influence of supraspi-

Table 1 - Effect of spinal cord contusion injury on the number of Fos protein-immunoreactive cells in the thoracic spinal cord and brain stem structures.

The quantitative analysis was performed in laminae I-II and lamina $X$ of the spinal cord and in the nucleus of the reticular formation, in the solitary tract nucleus of the brain stem, and in the area postrema. $\mathrm{N}=3$ animals/ group and 3 sections/region in each animal were analyzed to obtain each individual value. Results are reported as means \pm SEM. *Indicates significant difference between groups $(P<0.05$, t-test).

\begin{tabular}{lcccccc}
\hline & \multicolumn{2}{c}{ Spinal cord } & & \multicolumn{3}{c}{ Brain stem } \\
\cline { 2 - 3 } \cline { 5 - 7 } & Laminae I-II & Lamina X & & $\begin{array}{c}\text { Nucleus of the } \\
\text { reticular formation }\end{array}$ & $\begin{array}{c}\text { Solitary } \\
\text { tract nucleus }\end{array}$ & $\begin{array}{c}\text { Area } \\
\text { postrema }\end{array}$ \\
\hline Laminectomy group & $0.0 \pm 0.0$ & $6.0 \pm 6.0$ & & $44.0 \pm 9.0$ & $47.3 \pm 5.2$ & $61.0 \pm 29.0$ \\
Contusion group & $37.7 \pm 1.2^{*}$ & $15.0 \pm 3.2$ & & $70.7 \pm 15.9^{*}$ & $73.0 \pm 5.6^{*}$ & $226.0 \pm 16.5^{*}$
\end{tabular}


nal nervous centers. Our observations of Fos protein expression in the reticular formation, area postrema and solitary tract nuclei agree with this hypothesis. Also, our observations support the hypothesis of Lenz et al. (17) that the pathophysiology of spinal cord injury involves changes not only in the spinal cord but also in supraspinal levels of the neuroaxis. Ruggiero et al. (8), commented that sympathetic nerve activity is maintained after high spinal injury through circuits that remain in question. We conclude that brain stem neurons with sympathetic nerve-related activity are selectively activated by spinal cord contusion.

Traumatic or ischemic injury of the CNS triggers reactive biochemical variations, some of them being destructive and others neuroprotective (2,13). Munglani and Hunt (4) and Ikeda and Nakagawa (18) have suggested that proteins may be synthesized in response to injury and may participate in the neuronal response secondary to injury. Concerning the functional relevance of the in- duction of the c-Fos proto-oncogene-positive neurons after injury, the increased activity of the damaged neurons may be involved in neural changes after the lesion, although it is not possible to distinguish between regenerative and/or degenerative processes $(6,19)$.

The initial reports cited here only mentioned the detection of changes in neuronal phenotype and gene expression in spinal cord injuries. We believe that a complete quantitative evaluation of the functional state of supraspinal neurons with more accurate behavioral analysis should be performed for a better investigation and understanding of this process and of its possible interrelations with already known phenomena of spinal cord injury.

\section{Acknowledgments}

We are indebted to José Carlos de Aguiar, Eleni L.T. Gomes, Paulo Castania, and Célia Aparecida da Silva for excellent technical assistance.

\section{References}

1. Nicholls J \& Saunders N (1996). Regeneration of immature mammalian spinal cord after injury. Trends in Neurosciences, 19: 229-234.

2. Schwab ME \& Bartholdi D (1996). Degeneration and regeneration of axons in the lesioned spinal cord. Physiological Reviews, 76: 319-370.

3. J enkins R, Tetzlaff W \& Hunt SP (1993). Differential expression of immediate early genes in rubrospinal neurons following axotomy in the rat. European J ournal of Neuroscience, 5: 203-209.

4. Munglani R \& Hunt SP (1995). Proto-oncogenes: basic concepts and stimulation induced changes in spinal cord. In: Wiesenfeld-Hallin S, Sharma N \& Nyberg $F$ (Editors), Neuropeptides in the Spinal Cord. Fundamental and Clinical Aspects. Elsevier Science B.V., Amsterdam, 283298.

5. Hunt SP, Pini A \& Evan G (1987). Induction of c-fos-like protein in spinal cord neurons. Nature, 328: 632-634.

6. Smeyne RJ, Vendrell M, Hayward M,
Baker SJ , Miao GG, Schilling K, Robertson LM, Curran T \& Morgan JI (1993). Continuous c-fos expression precedes programmed cell death in vivo. Nature, 363 : 166-169.

7. Weiser M, Baker H, Wessel TC \& J oh TH (1993). Axotomy induced differential gene induction in neurons of the locus coeruleus and substantia nigra. Molecular Brain Research, 17: 319-327.

8. Ruggiero DA, Anwar M, Kim J, Gootman N \& Gootman PM (1997). Induction of cfos gene expression by spinal cord transection in the rat. Brain Research, 763: 21-29.

9. Bohlman $\mathrm{HH}$, Eugene B, Field $\mathrm{G} \&$ George $T$ (1981). Spinal cord monitoring of experimental incomplete spinal cord injury: A preliminary report. Spine, 6: 428-436.

10. Del Bel EA, Silveira MCL, Guimarães FS, Graeff FG \& Garcia-Cairasco N (1998). CFOS MRNA and protein distribution patterns in rat brain after restraint stress and pentylenetetrazol-induced seizures. Cellular and Molecular Neurobiology, 18: 339-
346.

11. Paxinos G \& Watson DV (1982). The Rat Brain in Stereotaxic Coordinates. Academic Press, New York.

12. Nadelhaf I \& Booth AM (1984). The location and morphology of preganglionic neurons and the distribution of visceral afferent from the rat pelvic nerve: a horseradish peroxidase study. J oumal of Comparative Neurology, 226: 238-245.

13. Taoka Y \& Okajima K (1998). Spinal cord injury in the rat. Progress in Neurobiology, 56: 341-358.

14. Yerzierski RP, Liu S, Ruenes KJ , Kajander KJ \& Brewer KL (1998). Excitotoxic spinal cord injury: behavioral and morphological characteristics of a central pain model. Pain, 75: 141-155.

15. Thompson FJ, Reier PJ, Lucas CC \& Parmer R (1992). Altered patterns of reflex excitability subsequent to contusion injury of the rat spinal cord. J ournal of Neurophysiology, 68: 1473-1486.

16. Krens NR \& Weaver LC (1996). Sprouting of primary afferent fibers after spinal cord 
transection. Abstracts of the Society for Neuroscience, 26th Annual Meeting, Washington, DC, Part 2: 1488.

17. Lenz FA, Tasker RR, DostrovskyJ O, Kwan HC, Gorecki J M, Hirayama T \& Murphy J T (1987). Abnormal single unit activity recorded in the somatosensory thalamus of a quadriplegic patient with central pain. Pain, 31: 225-236.

18. Ikeda S \& Nakagawa S (1998). Spinal cord transection induced c-Fos protein in rat motor cortex. Brain Research, 792: 164167.

19. Rhagupathy LJ , Rosemberg TK, McIntosh
TK \& Lucas J K (1996). Increased immunoreactivity of C-jun and jun-B but not jun$D$ in dying and dead neurons after physical injury. Abstracts of the Society for Neuroscience, 26th Annual Meeting, Washington, DC, Part 1: 21. 